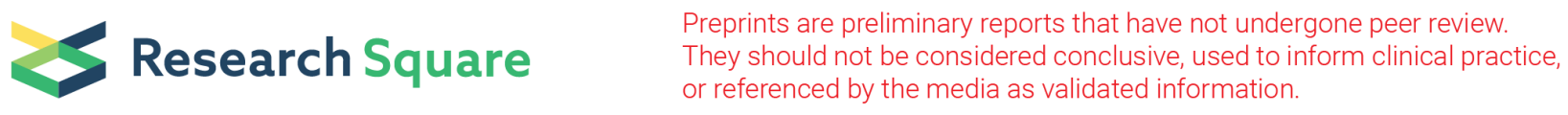

\title{
Health care costs and lost productivity costs related to excess weight in Belgium
}

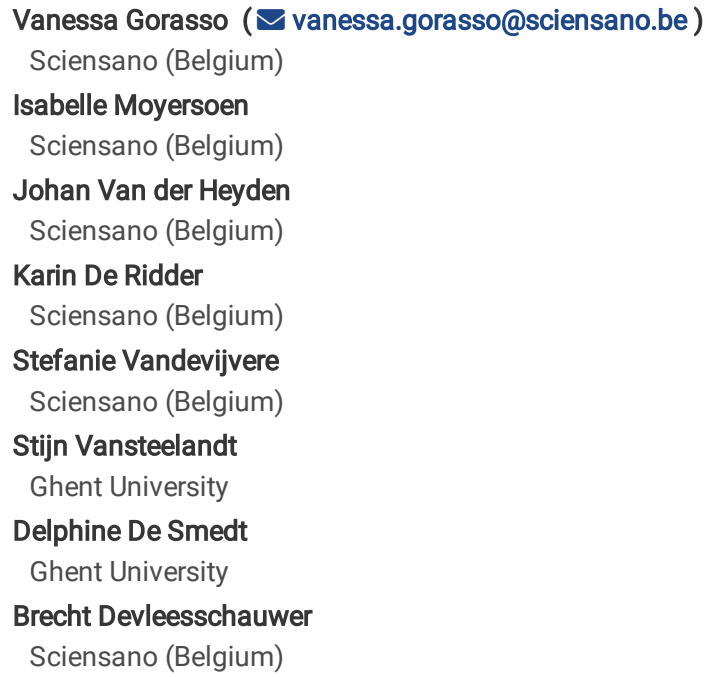




\section{Abstract \\ Background}

This study aimed to estimate annual health care and lost productivity costs associated with excess weight among the adult population in Belgium, using national health data.

\section{Methods}

Health care costs and costs of absenteeism were estimated using data from the Belgian national health interview survey (BHIS) 2013 linked with individual health insurance data (2013-2017). Average yearly health care costs and costs of absenteeism were assessed by body mass index (BMI) categories - i.e., underweight $\left(\mathrm{BMI}<18.5 \mathrm{~kg} / \mathrm{m}^{2}\right)$, normal weight $\left(18.5 \leq \mathrm{BMl}<25 \mathrm{~kg} / \mathrm{m}^{2}\right)$, overweight $\left(25 \leq \mathrm{BMl}<30 \mathrm{~kg} / \mathrm{m}^{2}\right)$ and obesity $\left(\mathrm{BMI} \geq 30 \mathrm{~kg} / \mathrm{m}^{2}\right)$. Health care costs were also analysed by type of cost (i.e. ambulatory, hospital, reimbursed medication). The cost attributable to excess weight and the contribution of various other chronic conditions to the incremental cost of excess weight were estimated using the method of recycled prediction (a.k.a. standardisation).

\section{Results}

According to BHIS $2013,34.7 \%$ and $13.9 \%$ of the Belgian adult population were respectively overweight or obese. They were mostly concentrated in the agegroup 35-65 years and had significantly more chronic conditions compared to the normal weight population. Average total healthcare expenses for overweight and obese people were significantly higher than those observed in the normal weight population.

\section{Background}

The sustained global increase of overweight and obesity over the last 40 years puts a heavy burden on the health system worldwide [1]. In 2019, excess weight was one of the top three risk factors in terms of attributable death and disability-adjusted life years (DALYs) and was increasing in exposure by more than $1 \%$ per year globally [2]. Cardiovascular disease, type 2 diabetes, kidney diseases and neoplasms account for about $90 \%$ of the excess weight-related DALYs globally [2]. Excess weight is strongly associated with the occurrence of chronic diseases, impaired health-related quality of life, increased health care and medication spending [3] [4] and a decreased workforce productivity [5].

Despite the disturbing figures in the global obesity prevalence and the related costs, no country or subpopulation was able yet to reverse the upward trend of obesity [1], [6]. Addressing the obesity pandemic requires a multi-sectoral approach across multiple areas of governance and well-defined programs on control and prevention. Along with epidemiological studies, cost of illness studies help to demonstrate the harmful effects of diseases in financial terms. This information, with cost-effectiveness studies, can then be used by policy makers to prioritise the allocation of resources to prevention, treatment and research [7].

In Belgium, as in many high-income countries, average body mass index (BMI) has increased over the past decades among both children and adults. According to the Belgian health examination survey (BHES), in 2018, more than half of the adult population was overweight and 16\% was obese [8]. The latest available cost data reported that in 2010, around $€ 600$ million was spent on medical care of obesity related pathologies [9]. In addition, based on a Markov decision-analytic model, a one unit BMI-reduction in the total overweight and obese Belgian population was estimated to result in a societal cost (direct and indirect) saving of $€ 2.8$ billion [10].

Considering the importance of this risk factor and the need for updated evidence, this study investigates the burden of excess weight including overweight and obesity among the adult population on annual health care costs and lost productivity costs in Belgium, and investigates to what extent differences in expenditures by BMI differ by socio-demographic characteristics and comorbidity burden.

\section{Methods}

\subsection{Data}

Individual participant health care costs related to obesity and overweight were obtained by linking two national databases, i.e. the Belgian Health Interview Survey (BHIS) 2013 and the national health insurance data compiled by the Intermutualistic Agency (IMA) 2013-2017. Linkage was performed by means of a National Registry Number. The BHIS was conducted between January and December 2013 among a representative sample of the Belgian population ( $\mathrm{N}$ = $10,828)$ and comprises data on health status and related health behaviour and determinants. Respondents were recruited following a multistage sampling design, as described in detail elsewhere [11]. Interviews were performed using a face-to-face paper and pencil interviewing, supplemented with a selfadministered questionnaire covering more sensitive topics [11]. Health insurance is compulsory in Belgium covering more than $99 \%$ of the population. The linked IMA database used for this study comprises aggregated reimbursed health care costs from 2013-2017 for all HIS participants including expenditures for 1) ambulatory care (pharmaceuticals excluded), 2) hospital care, and 3) reimbursed medicines purchased through public pharmacies. Available information on hospital care only included variable costs. However, in Belgium, the national health insurance also pays a fixed amount to the hospitals per admitted patient, depending on the type of hospital and treatment. Precise information on these costs was not directly available in the dataset. In order to estimate the fixed part of the total hospital care cost, the hospitalizations per patient per year were multiplied with the average annual $100 \%$ per diem cost 
publicly available by type of hospitalization (per diem costs available through: https://www.riziv.fgov.be/nl/themas/kost-terugbetaling/doorziekenfonds/verzorging-ziekenhuizen/Paginas/verpleegdagprijzen-ziekenhuizen.aspx).

The study included the adult population (age $\geq 18$ years) who reported weight and height and for whom linkage with health insurance data was possible and were continuously insured from 2013-2017 (latest linkage available). People who deceased during the study period (from their participation to the BHIS until 31/12/2017) were excluded. The final study sample comprised 7,633 participants (Fig. 1).

Health care costs were analysed by BMI category calculated from self-reported weight and height obtained from the BHIS using the classification recommended by the World Health Organization, i.e., underweight $\left(\mathrm{BMl}<18.5 \mathrm{~kg} / \mathrm{m}^{2}\right)$, normal weight $\left(18.5 \leq \mathrm{BMI} \leq 24.99 \mathrm{~kg} / \mathrm{m}^{2}\right)$, overweight $(25 \leq \mathrm{BMI}<30$ $\left.\mathrm{kg} / \mathrm{m}^{2}\right)$ and obese $\left(\mathrm{BMI} \geq 30 \mathrm{~kg} / \mathrm{m}^{2}\right.$ ) [12]. Socio-demographic variables taken into account included age, gender, household educational level (i.e. the highest educational level within the household), and income level (based on the calculated quintiles of the household income), as well as behavioural risk factors with respect to alcohol misuse, smoking, poor dietary quality and physical inactivity. Analyses also concerned information from the BHIS database on the prevalence of 23 major chronic conditions, i.e., asthma, chronic bronchitis, myocardial infarction, coronary heart disease, other serious heart disease, hypertension, high blood cholesterol, stroke, narrowing of blood vessels, arthritis (including rheumatoid arthritis and osteoarthritis), low back pain, neck pain, diabetes, allergy, stomach ulcer, cirrhosis of the liver, cancer, severe headache, urinary incontinence, serious gloom or depression, thyroid problems, and eye disease.

Absenteeism was reported in the BHIS as days absent from work during the 12 months prior to the BHIS interview queried by the following question: "Have you been absent from work during the past 12 months due to health problems? In doing so, take into account any conditions, injuries or other health problems you may have had and which resulted in an absence from work". Followed by the question: "How many days in total have you been absent from work for the past 12 months due to health problems? If you are unable to indicate this number of days correctly, please give an estimate.". The question was asked to working individuals only $(\mathrm{N}=3,857)$ - individuals that stated to have a paid job at the moment of the interview.

\subsection{Analysis}

Analyses were conducted in R 4.0.5 [13] taking the design of the survey into account. The sampling design included stratification at the level of the provinces and clustering at the household level, as described in Demarest et al [11]. Analysis of socio-demographic characteristics and healthcare costs per BMI category were performed using nominal logistic regression for comparison of proportions between BMI categories with normal weight as reference group. Confidence intervals $(\mathrm{Cl})$ were computed via the delta method, using the standard errors resulting from the survey analysis.

\subsubsection{Health care costs}

Overall health care costs and health care costs by type and by payment modality were calculated per BMI category. Univariate and multivariable regressions with negative binomial distribution and log link were used to explore the extent to which average yearly health care cost was associated with BMI category, socio-demographic characteristics and behavioural risk factors. The univariate model of health care cost in function of BMI-class allowed to estimate the unadjusted incremental health care costs and to evaluate statistical differences in average costs between underweight, overweight and obese individuals compared to normal weight individuals.

A "double-selection" approach was used for the selection of the variables to be included in the final multivariable model, based on backward elimination to identify significant variables at the $10 \%$ level [14]. The variables were identified in two steps, finding those that predict the dependent variable and those that predict the independent variables (BMI-class). The final linear regression included the covariates identified in either of the two steps. Candidate explanatory variables included age groups, gender, household educational level, household level of income and some behavioural risk factors such as smoking, alcohol misuse, unhealthy eating behaviour and physical inactivity. This use of double-selection is more likely to detect common causes of BMI-category and costs, and thereby results in more accurate inferences that also acknowledge the uncertainty in the selected variables.

\subsubsection{Indirect costs - Cost of absenteeism}

Cost of absenteeism was computed by multiplying the number of days absent from work by the national average labour cost per day. Using the costing year 2010 from Eurostat, the average Belgian labour cost per working day was estimated at €257 (monthly labour cost and assuming 18.8 working days per month (i.e., 52 weeks * 5 working days minus 24 days (legal holidays and agreed extra holidays) minus 10 public holidays) [18]. However, since respondents might have included weekend days in their answer, the total days absent from work was subtracted from the maximum number of working days per year, i.e., 226 days. If this difference was equal to or greater than zero, the answer was kept, else the maximum number of working days was used. The "double-selection" approach was performed also for the indirect costs, see above for more details.

\subsubsection{Attributable cost of excess weight status}

The final regression model allowed to estimate the adjusted attributable costs and associated uncertainties of overweight and obesity compared to normal weight. Incremental costs were estimated at the individual level using the method of recycled predictions (also known as direct standardisation or $\mathrm{g}$ computation) that allows to estimate the marginal effect from overweight and obesity on health care costs [15], [16]. The coefficients of the regression model were used to 1) predict health care costs for each respondent using the BMI from their reported weight and height; 2) predict health care cost assuming all respondents did have a normal BMI, keeping all other characteristics as observed; 3 ) calculate the individual incremental cost of obesity as the difference of an individual's predicted costs assuming they were obese or overweight and assuming they had normal weight; and 4) calculate the attributable cost of obesity as the population survey-weighted average of the individual incremental cost. In order to jointly reflect prediction and survey uncertainty, means and Cls by BMI-classes were computed via bootstrapping with 1000 replicates and 1000 Monte Carlo simulations drawn per replication (for the survey design), leading to $1000 * 1000$ interactions. In addition, total direct costs were calculated multiplying the average incremental cost by the proportion of individuals with 
overweight and obesity in the total adult population on the 1 st of January $2018(\mathrm{~N}=9,074,575)$ [17]. According to the BHIS2018, 33.4\% and 15.9\% of the Belgian adult population were respectively overweight and obese. Total indirect costs were calculated multiplying the average incremental absenteeism cost by the proportion of individuals with overweight and obesity in the total population with a paid job according to BHIS2013 ( $\mathrm{N}=3,906,170)$ [17], namely 33.9\% and $11.3 \%$ of the Belgian population with a paid job were respectively overweight and obese.

\subsubsection{Relative contribution of chronic conditions}

To investigate the relative importance of chronic conditions contributing to differences in health expenditure of persons with obesity and overweight compared to normal weight individuals, we evaluated how much of the attributable cost of excess weight can be attributed to each of 23 diseases. For this, we 1 ) extended the regression model for health care costs to also include the considered disease along with the covariates significant in a model with the disease as dependent variable; 2 ) used the model to predict health care cost assuming all respondents had a normal BMI, keeping all other characteristics (including disease status) as observed; 3) subtracted the predictions obtained in step 2 of the previous section from the obtained predictions; and 4) calculated how much of the attributable cost of obesity is due to the considered disease as the population survey-weighted average of the individual incremental cost obtained in the previous step, and dividing this by the average incremental cost of excess weight. For these analyses, the underweight population was omitted, considering that diseases related to underweight are commonly different from those related to excess weight. This method allowed to rank the diseases by their relative contribution to the incremental cost of obesity.

\section{Results}

Table 1 describes socio-demographic characteristics and number of chronic conditions of the study population by BMI category. In $2013,13.9 \%$ of the adult population aged 18 years and older was obese while $34.7 \%$ was overweight. The overweight individuals (excluding the population with obesity) comprised significantly more men than women and older than younger adults compared to the normal weight individuals. In the overweight and obese BMI category, there were significantly more participants with lower educational levels and lower income, compared to the normal weight BMI category. The results show that a higher BMI is significantly associated with the number of chronic conditions. The prevalence of respondents with 3 or more chronic conditions increased from $2 \%$ in normal weight individuals to $4 \%$ and $10 \%$ in overweight and obese participants respectively. 
Table 1

Socio-demographic characteristics by body mass index category, Belgian population $\geq 18$ years, BHIS 2013

\begin{tabular}{|c|c|c|c|c|c|c|c|c|c|c|}
\hline & \multicolumn{2}{|l|}{ Total } & \multicolumn{2}{|c|}{ Underweight } & \multicolumn{2}{|c|}{ Normal weight } & \multicolumn{2}{|c|}{ Overweight } & \multicolumn{2}{|l|}{ Obese } \\
\hline & $\mathrm{N}^{(1)}$ & $\%(2)$ & $\mathrm{N}$ & $\%$ & $\mathrm{~N}$ & $\%$ & $\mathrm{~N}$ & $\%$ & $\mathrm{~N}$ & $\%$ \\
\hline Total & 7,633 & 100 & 222 & 2.8 & 3,692 & 48.6 & 2,624 & 34.7 & 1,095 & 13.9 \\
\hline \multicolumn{11}{|l|}{ Gender } \\
\hline Men & 3,613 & 48.3 & 51 & 25.0 & 1,540 & 43.0 & 1,510 & 58.0 & 512 & 46.9 \\
\hline Women & 4,020 & 51.7 & 171 & $75.0 *$ & 2,152 & 57.0 & 1,114 & $42.0 *$ & 583 & 53.1 \\
\hline \multicolumn{11}{|l|}{ Age (in years) } \\
\hline $18-34$ & $1,978 \quad 27$ & & 113 & $64.2^{\star}$ & 1,263 & 33.9 & 446 & $18.1^{*}$ & 156 & $15.6^{*}$ \\
\hline $35-65$ & 3,945 & 52 & 74 & $25.1^{*}$ & 1,781 & 49.8 & 1,461 & $55.7^{*}$ & 629 & $53.3^{*}$ \\
\hline$\geq 66$ years & 1,710 & 21 & 35 & $10.7 *$ & 648 & 16.3 & 717 & $26.2^{*}$ & 310 & $28.1^{\star}$ \\
\hline \multicolumn{11}{|l|}{ Household education } \\
\hline No diploma/primary & 779 & 9.3 & 19 & 4.4 & 253 & 5.6 & 311 & $11.0 *$ & 196 & $19.0 *$ \\
\hline Lower secondary & 1,075 & 13.1 & 33 & 13.7 & 447 & 11.1 & 393 & $14.0^{*}$ & 202 & $18.0^{*}$ \\
\hline Higher secondary & 2,486 & 34.7 & 70 & 37.8 & 1,159 & 34.5 & 870 & 34.0 & 387 & $36.6^{*}$ \\
\hline Higher education & 3,191 & 42.9 & 99 & 44.1 & 1,780 & 48.9 & 1,014 & 41.0 & 298 & 26.4 \\
\hline \multicolumn{11}{|l|}{ Household income } \\
\hline Quintile 1 & 1,398 & 16.9 & 55 & 24.5 & 589 & 14.8 & 489 & 16.9 & 265 & $22.5^{*}$ \\
\hline Quintile 2 & 1,136 & 16.1 & 30 & 13.5 & 505 & 14.2 & 396 & 16.2 & 205 & $22.6^{*}$ \\
\hline Quintile 3 & 1,347 & 20.9 & 35 & 20.4 & 652 & 22.0 & 466 & 20.5 & 194 & 18.1 \\
\hline Quintile 4 & 1,357 & 22.2 & 36 & 22.3 & 688 & 22.8 & 473 & 22.3 & 160 & 19.8 \\
\hline Quintile 5 & 1,496 & 23.9 & 41 & 19.3 & 792 & 26.1 & 500 & 24.1 & 163 & 17.0 \\
\hline \multicolumn{11}{|c|}{ Number of chronic conditions } \\
\hline None & 4,836 & 64 & 163 & 79 & 2,716 & 75 & 1,507 & 58 & 450 & 42 \\
\hline 1 & 1,738 & 22 & 36 & 14 & 669 & 18 & 697 & $26^{*}$ & 336 & $30 *$ \\
\hline 2 & 740 & 9 & 19 & 5 & 232 & 6 & 293 & $11^{*}$ & 196 & $17 *$ \\
\hline 3 or more & 315 & 4 & 4 & 1 & 71 & 2 & 127 & $4^{\star}$ & 113 & $10 *$ \\
\hline
\end{tabular}

1. Survey weighted prevalence

* Prevalence significantly different $(p<0.001)$ relative to normal BMI-class

\subsection{Health care cost per BMI category}

Table 2 shows the average yearly health care costs per capita from 2013-2017. The average yearly total health care cost increased from $€ 2,246$ per capita in normal weight individuals to $€ 3,475$ and $€ 4,288$ per capita in overweight and obese individuals respectively. In underweight individuals the average health care expenditure was $€ 3,387$ per capita per year. Ambulatory care was $58 \%$ of total health care cost while $30 \%$ of the costs were for hospital care and $9.5 \%$ for reimbursed medicines obtained through pharmacies. 
Table 2

Mean annual health expenses (in euro) per capita in function of body mass index categories, Belgian population $\geq 18$ years, BHIS $2013-$ IMA

\begin{tabular}{|c|c|c|c|c|c|c|c|c|c|c|}
\hline Health expenses & \multicolumn{2}{|l|}{ Total } & \multicolumn{2}{|c|}{$\begin{array}{l}\text { Underweight } \\
\mathrm{BMI}<18\end{array}$} & \multicolumn{2}{|c|}{$\begin{array}{l}\text { Normal weight } \\
18 \leq \mathrm{BMI}<24.5\end{array}$} & \multicolumn{2}{|c|}{$\begin{array}{l}\text { Overweight } \\
25 \leq \mathrm{BMl}<29.5\end{array}$} & \multicolumn{2}{|c|}{$\begin{array}{l}\text { Obese } \\
\text { BMI } \geq 30\end{array}$} \\
\hline Ambulatory care & 1,766 & 58 & 1,585 & $1,203-1,968$ & 1,413 & $1,288-1,538$ & 2,061 & $1,814-2,307$ & 2,299 & $2,054-2,545$ \\
\hline Hospital care & 932 & 30 & 1,184 & $616-1,707$ & 741 & $616-865$ & 1,004 & $870-1,128$ & 1,370 & $1,115-1,625$ \\
\hline Not specified & 77 & 2.5 & 87 & $56-118$ & 63 & $52-74$ & 83 & $73-94$ & 107 & $88-126$ \\
\hline Total health care cost & 3,066 & 100 & 3,038 & $2,252-3,824$ & 2,426 & $2,192-2,660$ & 3,475 & $3,133-3,818$ & 4,288 & $3,803-4,772$ \\
\hline
\end{tabular}

\subsection{Incremental cost of overweight and obesity at national level and per capita}

The unadjusted negative binomial regression model allowed to estimate the incremental costs of overweight and obese individuals versus normal weight individuals. The average yearly health care cost of overweight and obese individuals were significantly higher (i.e. $43 \%$ and $77 \%$ higher $-p<0.001$ ) compared to normal weight individuals (Table 3). Based on the method of recycled predictions, the mean incremental cost of overweight and obesity were $€ 1,046$ [Cl: $677-1,445]$ and $€ 1,870$ [Cl: 1,388-2,407] per capita, respectively, leading to a national incremental cost of $€ 3,170,527,646$ for the overweight population and $€ 2,698,190,204$ for the obese population.

Table 3

Unadjusted health care costs in function of body mass index classes, Belgian population $\geq 18$ years, BHIS 2013 - IMA2013-2017 ( $N=7,633)$

\begin{tabular}{|c|c|c|c|c|c|}
\hline BMI categories & $\mathrm{RR}^{(1)}$ & Standard error & P-value & $95 \% \mathrm{Cl}$ & Mean incremental cost ${ }^{(2)}$ \\
\hline Underweight & 1.25 & 0.17 & 0.111 & $0.91-1.65$ & 627 \\
\hline Normal weight (reference) & 1 & - & - & - & - \\
\hline Overweight & 1.43 & 0.10 & $<0.001$ & $1.25-1.64$ & 1,046 \\
\hline Obese & 1.77 & 0.13 & $<0.001$ & $1.54-2.03$ & 1,870 \\
\hline Constant & 2,426 & 119 & $<0.001$ & $2,203-2,672$ & \\
\hline
\end{tabular}

(1) RR = expected value of the coefficient with normal weight as reference category

(2) Based on the method of recycled predictions

Since increased health expenses in overweight and obese individuals compared to normal weight individuals are also related to socio-demographic factors and chronic health conditions, results presented in Table 3 needed to be adjusted. Univariate regression analysis of health care cost in function of BMI and each of the candidate predictors revealed that age, gender, household educational level and lack of physical activity likely had a confounding effect on BMI-related health care costs $(p<0.01)$. In addition, the multinomial regression of BMI categories as function of the candidate covariates revealed that the same predictors were significant (see Appendix Table 7). Moreover, considering that the average incremental cost of underweight was not statistically different from the normal weight one, the underweight population $(\mathrm{N}=222)$ was excluded from further analysis.

The final multivariable model included age, gender, educational level and lack of physical activity as independent variables (Table 4). The high nonresponse rate on the physical activity questions in the BHIS resulted in a reduced sample size $(\mathrm{N}=4,624 ; 60.6 \%)$. Since lack of physical activity is an important behavioural risk factor for chronic diseases, this indicator was kept as possible confounder in the multivariable model even if its inclusion would lead to a reduced sample size. In addition, sociodemographic characteristics of the reduced sample didn't differ much from the original sample (see Appendix Table 6). Based on this model, the adjusted mean incremental cost for overweight and obesity was $€ 651$ [95\% Cl: $€ 144-€ 1,084]$ and $€ 1,015$ [95\% Cl: $€ 343-€ 1,697]$ per capita respectively. At national level, the adjusted incremental health care cost for the overweight population was $€ 1,864,464,355$ and $€ 1,464,742,302$ for the obese population leading to a total incremental cost for Belgium of $€ 3,329,206,657$ related to excess body weight. 
Table 4

Health care costs in function of body mass index categories adjusted for age, gender, household educational level and lack of physical activity, Belgian population $\geq 18$ years - underweight population was excluded, BHIS 2013 - IMA2013-

\begin{tabular}{|c|c|c|c|c|c|}
\hline & RR & Standard error & P-value & $95 \% \mathrm{Cl}$ & Mean incremental cost \\
\hline \multicolumn{6}{|l|}{ BMI categories } \\
\hline Normal weight (reference) & 1 & - & - & - & - \\
\hline Overweight & 1.24 & 0.11 & 0.010 & $1.05-1.47$ & 651 \\
\hline Obese & 1.36 & 0.14 & 0.003 & $1.11-1.65$ & 1,015 \\
\hline \multicolumn{6}{|l|}{ Age groups } \\
\hline $18-34$ years (reference) & 1 & - & - & - & \\
\hline $35-64$ years & 1.44 & 0.20 & 0.009 & $1.09-1.89$ & \\
\hline$\geq 65$ years & 2.62 & 0.41 & $<0.001$ & $1.93-3.55$ & \\
\hline \multicolumn{6}{|l|}{ Gender } \\
\hline Male (reference) & 1 & - & - & - & \\
\hline Female & 1.02 & 0.07 & 0.807 & $0.88-1.17$ & \\
\hline \multicolumn{6}{|l|}{ Household educational level } \\
\hline No diploma or primary education & 1.62 & 0.25 & 0.002 & $1.20-2.18$ & \\
\hline Lower secondary education & 1.48 & 0.16 & $<0.001$ & $1.21-1.82$ & \\
\hline Higher secondary education & 1.13 & 0.09 & 0.125 & $0.97-1.33$ & \\
\hline Higher education (reference) & 1 & - & - & - & \\
\hline \multicolumn{6}{|l|}{ At risk due to lack of physical activity } \\
\hline Yes & 1.37 & 0.12 & $<0.001$ & $1.15-1.62$ & \\
\hline No (reference) & 1 & - & - & - & \\
\hline Constant & 1,164 & 152 & $<0.001$ & $901-1,503$ & \\
\hline
\end{tabular}


Table 7

Multinomial regression of body mass index classes (normal weight category as reference) in function of age, gender, educational level and lack of physical activity, Belgian population $\geq 18$ years - underweight population was excluded, BHIS2013-IMA2013-2017 - the model includes the confounders that were significant after backwards stepwise elimination $(N=4,504)$

\begin{tabular}{|c|c|c|c|c|c|c|}
\hline & \multicolumn{3}{|l|}{ Overweight } & \multicolumn{3}{|l|}{ Obesity } \\
\hline & Coefficient & Std. error & P-value & Coefficient & Std. error & P-value \\
\hline \multicolumn{7}{|l|}{ Age-groups } \\
\hline \multicolumn{7}{|l|}{ 18-34 years (base) } \\
\hline $35-64$ years & 0.56 & 0.12 & $<0.001$ & 0.86 & 0.19 & \\
\hline$\geq 65$ years & 0.85 & 0.14 & $<0.001$ & 0.69 & 0.23 & \\
\hline \multicolumn{7}{|l|}{ Gender } \\
\hline \multicolumn{7}{|l|}{ Male (base) } \\
\hline Female & -0.74 & 0.08 & $<0.001$ & -0.22 & 0.14 & $>0.100$ \\
\hline \multicolumn{7}{|l|}{ Educational level } \\
\hline \multicolumn{7}{|c|}{ No diploma or primary education (base) } \\
\hline Lower secondary education & 0.50 & 0.23 & 0.030 & -0.75 & 0.26 & 0.006 \\
\hline Higher secondary education & -0.72 & 0.20 & $<0.001$ & -1.20 & 0.25 & $<0.001$ \\
\hline Higher education & -0.85 & 0.19 & $<0.001$ & -1.68 & 0.29 & \\
\hline \multicolumn{7}{|c|}{ At risk due to lack of physical activity } \\
\hline Yes & 0.34 & 0.10 & 0.001 & 0.80 & 0.16 & $<0.001$ \\
\hline \multicolumn{7}{|l|}{ No (base) } \\
\hline Constant & 0.19 & 0.22 & $>0.100$ & -0.84 & 0.26 & 0.002 \\
\hline
\end{tabular}

\subsection{Disease associated health care cost related to overweight and obesity}

Figure 2 presents the relative contribution of chronic conditions to the incremental direct costs of overweight and obesity among adults in Belgium. Appendix Table 8 shows the details of the fitted models of each chronic condition based on the significant confounders. 
Table 8

Chronic disease in function of available confounders - coefficient (standard error), Belgian population $\geq 18$ years - underweight population was excluded, BHIS 2013-IMA2013-2017 - the model includes the confounders that were significant after backwards stepwise elimination

\begin{tabular}{|c|c|c|c|c|c|c|c|c|c|c|}
\hline Allergy & Asthma & Cancer & Cirnhosis & $\begin{array}{l}\text { Coronary } \\
\text { heart } \\
\text { disease }\end{array}$ & $\begin{array}{l}\text { Chronic } \\
\text { bronchitis } \\
\text { and } \\
\text { COPD }\end{array}$ & Diabetes & $\begin{array}{l}\text { Eye } \\
\text { disease }\end{array}$ & $\begin{array}{l}\text { High } \\
\text { cholesterol }\end{array}$ & $\begin{array}{l}\text { Hyper- } \\
\text { tension }\end{array}$ & $\begin{array}{l}\text { Lower } \\
\text { Back } \\
\text { Pain }\end{array}$ \\
\hline
\end{tabular}

\section{Age-groups}

$18-34$ years

(base)

35-64 years

285

$\geq 65$ years

2.85

16.7

$1.80 \quad 1.05$

1.05

1.41

.41

1.34

1.34
$(0.35)$

$1.72(0.26)$

2.46

1.88

$2.18 \quad 3.35$

1.58
$(0.50)$

-0.57
$(0.30)$

$4.13 \quad 16.5$

3.06

$(0.43)$

3.35
$(0.32)$

$2.77(0.27) \quad 3.25$

$(0.30)$

$(0.36)$

(0.40)

17.88

Gender

Male (base)

$0.32 \quad 0.71$

0.71
$(0.26)$

$-0.37$

(0.19)

0.63

(010)

(0.26)

\section{Educational}

level

No diploma

or primary

education

(base)

Lower

secondary

education

Higher

secondary

education

-1.05
$(0.27)$

(0.27)

$-1.14$

(0.26)

-0.53
$(0.23)$

$-0.41$

$(0.22)$

Higher

education

(0.26)

Income

Quantile 1

(base)

Quantile 2

$\begin{array}{ll} & -1.37 \\ & (0.78) \\ -0.71 & -2.48\end{array}$

Quantile 3

(0.33)

(0.75)

Quantile $4 \quad 0.51$

$-0.61$

(0.18)

(0.36)

Quantile $5 \quad 0.45$

(0.18)

$-1.02$

$(0.37)$

$-2.34$

$(0.72)$

\section{Nationality}

Belgian

(base)

Non-Belgian

$-16.45$

Non-EU -13.54

(0.31)

\section{At risk due to}

lack of

physical

activity

Yes

(0.20)

0.73

$(0.73)$

1.25

$(0.40)$

0.87

$(0.25)$

0.48

$(0.20)$

$\begin{array}{lll}0.34(0.12) & 0.35 & 0.24 \\ & (0.16) & (0.12)\end{array}$

No (base)

Daily intake

of sugared

drinks 


\begin{tabular}{|c|c|c|c|c|c|c|c|c|c|c|c|c|}
\hline & Allergy & Asthma & Cancer & Cirrhosis & $\begin{array}{l}\text { Coronary } \\
\text { heart } \\
\text { disease }\end{array}$ & $\begin{array}{l}\text { Chronic } \\
\text { bronchitis } \\
\text { and } \\
\text { COPD }\end{array}$ & Diabetes & $\begin{array}{l}\text { Eye } \\
\text { disease }\end{array}$ & $\begin{array}{l}\text { High } \\
\text { cholesterol }\end{array}$ & $\begin{array}{l}\text { Hyper- } \\
\text { tension }\end{array}$ & $\begin{array}{l}\text { Lower } \\
\text { Back } \\
\text { Pain }\end{array}$ & $\begin{array}{l}\text { Myocardial } \\
\text { infarction }\end{array}$ \\
\hline Yes & $\begin{array}{l}0.28 \\
(0.13)\end{array}$ & & & $\begin{array}{l}-2.54 \\
(1.04)\end{array}$ & & & $\begin{array}{l}-0.60 \\
(0.28)\end{array}$ & & & $\begin{array}{l}-0.38 \\
(0.17)\end{array}$ & & \\
\hline \multicolumn{13}{|l|}{ No (base) } \\
\hline \multicolumn{13}{|c|}{$\begin{array}{l}\text { Over- } \\
\text { consumption } \\
\text { of alcohol }\end{array}$} \\
\hline Yes & & & & & & & & & $0.50(0.21)$ & $\begin{array}{l}0.74 \\
(0.20)\end{array}$ & & \\
\hline \multicolumn{13}{|l|}{ No (base) } \\
\hline \multicolumn{13}{|c|}{$\begin{array}{l}\text { Heavy daily } \\
\text { smoking }\end{array}$} \\
\hline Yes & & & & $\begin{array}{l}1.01 \\
(0.59)\end{array}$ & & $\begin{array}{l}1.26 \\
(0.37)\end{array}$ & & & & & & \\
\hline \multicolumn{13}{|l|}{ No (base) } \\
\hline Constant & $\begin{array}{l}-2.26 \\
(0.17)\end{array}$ & & $\begin{array}{l}-7.58 \\
(0.61)\end{array}$ & $\begin{array}{l}-21.51 \\
(0.17)\end{array}$ & $\begin{array}{l}-7.11 \\
(0.99)\end{array}$ & & $\begin{array}{l}-3.65 \\
(0.48)\end{array}$ & $\begin{array}{l}-4.73 \\
(0.35)\end{array}$ & $\begin{array}{l}-3.46 \\
(0.25)\end{array}$ & $\begin{array}{l}-3.94 \\
(0.39)\end{array}$ & $\begin{array}{l}-3.36 \\
(0.37)\end{array}$ & \\
\hline
\end{tabular}


Table 8

(continued)

\begin{tabular}{|c|c|c|c|c|c|c|c|c|c|c|}
\hline & $\begin{array}{l}\text { Narrowing } \\
\text { blood-vessels }\end{array}$ & $\begin{array}{l}\text { Neck } \\
\text { pain }\end{array}$ & $\begin{array}{l}\text { Other serious } \\
\text { heart disease }\end{array}$ & $\begin{array}{l}\text { Serious } \\
\text { gloom }\end{array}$ & $\begin{array}{l}\text { Severe } \\
\text { headache }\end{array}$ & $\begin{array}{l}\text { Stomach } \\
\text { ulcer }\end{array}$ & Stroke & Arthritis & Thyroid & $\begin{array}{l}\text { Urinary } \\
\text { incontinence }\end{array}$ \\
\hline \multicolumn{11}{|l|}{ Age-groups } \\
\hline \multicolumn{11}{|l|}{$18-34$ years (base) } \\
\hline $35-64$ years & $1.98(1.03)$ & $\begin{array}{l}1.51 \\
(0.17)\end{array}$ & $2.07(0.67)$ & $\begin{array}{l}1.13 \\
(0.24)\end{array}$ & & $\begin{array}{l}1.28 \\
(0.37)\end{array}$ & $\begin{array}{l}17.04 \\
(0.29)\end{array}$ & $\begin{array}{l}1.71 \\
(0.21)\end{array}$ & $\begin{array}{l}0.90 \\
(0.19)\end{array}$ & $3.11(0.94)$ \\
\hline$\geq 65$ years & $3.06(1.03)$ & $\begin{array}{l}1.89 \\
(0.18)\end{array}$ & $3.19(0.67)$ & $\begin{array}{l}0.60 \\
(0.32)\end{array}$ & & $\begin{array}{l}1.69 \\
(0.40)\end{array}$ & $\begin{array}{l}17.50 \\
(0.34)\end{array}$ & $\begin{array}{l}2.80 \\
(0.21)\end{array}$ & $\begin{array}{l}1.27 \\
(0.20)\end{array}$ & $3.98(0.94)$ \\
\hline \multicolumn{11}{|l|}{ Gender } \\
\hline \multicolumn{11}{|l|}{ Male (base) } \\
\hline Female & & $\begin{array}{l}0.55 \\
(0.10)\end{array}$ & $-0.57(0.32)$ & $\begin{array}{l}0.47 \\
(0.18)\end{array}$ & $\begin{array}{l}1.18 \\
(0.13)\end{array}$ & & & $\begin{array}{l}0.60 \\
(0.08)\end{array}$ & $\begin{array}{l}1.79 \\
(0.16)\end{array}$ & $0.58(0.24)$ \\
\hline \multicolumn{11}{|l|}{ Educational level } \\
\hline \multicolumn{11}{|l|}{$\begin{array}{l}\text { No diploma or primary } \\
\text { education (base) }\end{array}$} \\
\hline $\begin{array}{l}\text { Lower secondary } \\
\text { education }\end{array}$ & & & & & & & & $\begin{array}{l}-0.33 \\
(0.16)\end{array}$ & & $-0.71(0.37)$ \\
\hline $\begin{array}{l}\text { Higher secondary } \\
\text { education }\end{array}$ & & & & & & & & $\begin{array}{l}-0.33 \\
(0.14)\end{array}$ & & $-1.03(0.37)$ \\
\hline Higher education & & & & $\begin{array}{l}-0.67 \\
(0.31)\end{array}$ & & & & $\begin{array}{l}-0.75 \\
(0.14)\end{array}$ & & $-1.76(0.40)$ \\
\hline \multicolumn{11}{|l|}{ Income } \\
\hline \multicolumn{11}{|l|}{ Quantile 1 (base) } \\
\hline \multicolumn{11}{|l|}{ Quantile 2} \\
\hline \multicolumn{11}{|l|}{ Quantile 3} \\
\hline Quantile 4 & & & & & & $\begin{array}{l}-0.64 \\
(0.35)\end{array}$ & & & & \\
\hline Quantile 5 & & & & $\begin{array}{l}-0.97 \\
(0.35)\end{array}$ & & $\begin{array}{l}-1.21 \\
(0.42)\end{array}$ & $\begin{array}{l}-1.64 \\
(0.80)\end{array}$ & & & \\
\hline \multicolumn{11}{|l|}{ Nationality } \\
\hline \multicolumn{11}{|l|}{ Belgian (base) } \\
\hline Non-Belgian & $-1.50(0.81)$ & & $-1.21(0.63)$ & & & & $\begin{array}{l}-1.82 \\
(1.05)\end{array}$ & & & $-2.23(0.83)$ \\
\hline Non-EU & $0.20(0.64)$ & & $0.29(0.62)$ & & $\begin{array}{l}1.16 \\
(0.35)\end{array}$ & & $\begin{array}{l}-17.03 \\
(0.37)\end{array}$ & & & \\
\hline \multicolumn{11}{|l|}{$\begin{array}{l}\text { At risk due to lack of } \\
\text { physical activity }\end{array}$} \\
\hline Yes & $0.87(0.30)$ & & $0.62(0.31)$ & $\begin{array}{l}0.40 \\
(0.20)\end{array}$ & & & $\begin{array}{l}1.37 \\
(0.42)\end{array}$ & & & $1.10(0.25)$ \\
\hline \multicolumn{11}{|l|}{ No (base) } \\
\hline \multicolumn{11}{|l|}{$\begin{array}{l}\text { Daily intake of } \\
\text { sugared drinks }\end{array}$} \\
\hline Yes & & $\begin{array}{l}0.22 \\
(0.11)\end{array}$ & & & $\begin{array}{l}0.42 \\
(0.15)\end{array}$ & & & & & \\
\hline \multicolumn{11}{|l|}{ No (base) } \\
\hline \multicolumn{11}{|l|}{$\begin{array}{l}\text { Over-consumption of } \\
\text { alcohol }\end{array}$} \\
\hline Yes & & & $-1.10(0.63$ & $\begin{array}{l}0.65 \\
(0.33)\end{array}$ & & & & & & $-1.21(0.50)$ \\
\hline \multicolumn{11}{|l|}{ No (base) } \\
\hline Heavy daily smoking & & & & & & & & & & \\
\hline
\end{tabular}




\begin{tabular}{|c|c|c|c|c|c|c|c|c|c|c|}
\hline & $\begin{array}{l}\text { Narrowing } \\
\text { blood-vessels }\end{array}$ & $\begin{array}{l}\text { Neck } \\
\text { pain }\end{array}$ & $\begin{array}{l}\text { Other serious } \\
\text { heart disease }\end{array}$ & $\begin{array}{l}\text { Serious } \\
\text { gloom }\end{array}$ & $\begin{array}{l}\text { Severe } \\
\text { headache }\end{array}$ & $\begin{array}{l}\text { Stomach } \\
\text { ulcer }\end{array}$ & Stroke & Arthritis & Thyroid & $\begin{array}{l}\text { Urinary } \\
\text { incontinence }\end{array}$ \\
\hline Yes & & & & $\begin{array}{l}0.54 \\
(0.27)\end{array}$ & $\begin{array}{l}0.62 \\
(0.22)\end{array}$ & $\begin{array}{l}0.57 \\
(0.34)\end{array}$ & & & & \\
\hline \multicolumn{11}{|l|}{ No (base) } \\
\hline Constant & $-6.53(1.04)$ & $\begin{array}{l}-3.70 \\
(0.17)\end{array}$ & $-6.16(0.67)$ & $\begin{array}{l}-3.29 \\
(0.38)\end{array}$ & $\begin{array}{l}-3.23 \\
(0.12)\end{array}$ & $\begin{array}{l}-4.62 \\
(0.41)\end{array}$ & $\begin{array}{l}-20.90 \\
(0.43)\end{array}$ & $\begin{array}{l}-3.85 \\
(0.26)\end{array}$ & $\begin{array}{l}-4.77 \\
(0.21)\end{array}$ & $-6.42(1.00)$ \\
\hline
\end{tabular}

Twenty out of twenty-three chronic conditions included in the regression model had a positive contribution to the incremental cost of overweight and obesity (eye disease, coronary heart disease and narrowing of blood vessels showed a negative attributable cost) - see Appendix Table 10. Costs attributable to hypertension were by far the highest among all considered chronic conditions. The second highest relative cost was attributed to high cholesterol followed by serious gloom and depression.

\subsection{Cost of absenteeism}

3,857 individuals were identified as the adult working population and included within the analysis (50.5\% of the sample included in the previous analysis). The mean incremental cost of absenteeism in underweight and overweight individuals was $€ 360$ and $€ 242$ per capita respectively but did not differ significantly from zero. However, the cost increases significantly to $€ 2,015$ [95\% Cl: $€ 179-€ 4,336]$ per capita in obese individuals. Based on the method of recycled predictions, productivity loss poses an incremental cost to the society of $€ 320,082,750$ and of $€ 889,469,387$ within the population with overweight and obesity respectively. The results for the adjusted model can be found in Appendix Table 9.

Table 5

Unadjusted costs of absenteeism in function of BMI-classes, Belgian working population $\geq 18$ years, BHIS 2013 ( $N=$

\begin{tabular}{|llllll|}
\hline BMl-classes & RR & Std. error & P-value & $95 \%$ Cl & Mean incremental cost per capita \\
\hline Underweight & 1.19 & 0.48 & 0.671 & $(0.54-2.63)$ & 360 \\
\hline Normal weight (reference) & 1 & - & - & - & - \\
\hline Overweight & 1.11 & 0.16 & 0.472 & $(0.83-1.48)$ & 242 \\
\hline Obese & 1.87 & 0.48 & 0.015 & $(1.13-3.09)$ & 2,015 \\
\hline Constant & 2,359 & 228 & $<0.001$ & $(1,952-2,851)$ & \\
\hline RR = expected value of the coefficient with normal weight as reference category; Cl: confidence intervals
\end{tabular}


Table 9

Cost of absenteeism in function of body mass index classes adjusted for age, gender, educational level, nationality, lack of physical activity, tobacco use and daily intake of sugared drinks - Belgian population $\geq 18$ years, BHIS 2013-IMA2013-2017 ( $\mathrm{N}=2,480)$

\begin{tabular}{|c|c|c|c|c|c|}
\hline & IRR & Std. error & P-value & $95 \% \mathrm{Cl}$ & Mean incremental cost \\
\hline \multicolumn{6}{|l|}{ BMl-classes } \\
\hline Underweight & 1.27 & 0.60 & $>0.100$ & $0.50-3.22$ & 718 \\
\hline Normal weight (base) & 1 & & & & \\
\hline Overweight & 1.30 & 0.21 & $>0.100$ & $0.95-1.79$ & 650 \\
\hline Obese & 1.78 & 0.62 & 0.097 & $0.90-1.52$ & 1,888 \\
\hline \multicolumn{6}{|l|}{ Age-groups } \\
\hline 18-34 years (base) & 1 & & & & \\
\hline $35-64$ years & 0.94 & 0.20 & $>0.100$ & $0.62-1.43$ & \\
\hline$\geq 65$ years & 0.28 & 0.22 & $>0.100$ & $0.06-1.30$ & \\
\hline \multicolumn{6}{|l|}{ Gender } \\
\hline Male (base) & 1 & & & & \\
\hline Female & 1.90 & 0.33 & $<0.001$ & $1.35-2.66$ & \\
\hline \multicolumn{6}{|l|}{ Educational level } \\
\hline No diploma or primary education & 1.67 & 0.71 & $>0.100$ & $0.72-3.83$ & \\
\hline Lower secondary education & 1.01 & 0.29 & $>0.100$ & $0.57-1.79$ & \\
\hline Higher secondary education & 1.25 & 0.24 & $>0.100$ & $0.86-1.81$ & \\
\hline Higher education (base) & 1 & & & & \\
\hline \multicolumn{6}{|l|}{ At risk due to lack of physical activity } \\
\hline Yes & 1.19 & 0.26 & $>0.100$ & $0.77-1.83$ & \\
\hline No (base) & 1 & & & & \\
\hline \multicolumn{6}{|l|}{ Nationality } \\
\hline \multicolumn{6}{|l|}{ Belgian (base) } \\
\hline Non-Belgian & 0.58 & 0.18 & 0.081 & $0.32-1.07$ & \\
\hline Non-EU & 0.95 & 0.45 & $>0.100$ & $0.37-2.41$ & \\
\hline \multicolumn{6}{|l|}{ Heavy daily smoking } \\
\hline Yes & 1.36 & 0.40 & $>0.100$ & $0.76-2.43$ & \\
\hline No (base) & 1 & & & & \\
\hline \multicolumn{6}{|l|}{ Daily intake of sugared drinks } \\
\hline Yes & 1.21 & 0.22 & $>0.100$ & $0.85-1.73$ & \\
\hline No (base) & 1 & & & & \\
\hline Constant & 1,414 & 295 & $<0.001$ & $939-2,128$ & \\
\hline
\end{tabular}




\begin{tabular}{|c|c|c|}
\hline Disease & Relative contribution to the direct cost & Relative contribution to the cost of absenteeism \\
\hline Hypertension & 12.85 & 5.50 \\
\hline High cholesterol & 6.68 & 2.94 \\
\hline Serious gloom or depression & 6.39 & 2.25 \\
\hline Arthritis & 4.95 & 10.00 \\
\hline Urinary incontinence & 4.32 & 0.26 \\
\hline Stomach ulcer & 4.22 & 0.09 \\
\hline Other serious heart disease & 3.77 & 2.54 \\
\hline COPD & 3.55 & 2.79 \\
\hline Diabetes & 3.18 & -0.31 \\
\hline Myocardial infarction & 2.60 & -0.60 \\
\hline Low back pain & 2.48 & 5.13 \\
\hline Stroke & 2.39 & 3.78 \\
\hline Cirmosis of liver & 2.20 & 0.56 \\
\hline Allergy & 1.99 & -1.05 \\
\hline Cancer & 1.37 & -2.70 \\
\hline Severe headache & 1.05 & -2.01 \\
\hline Neck pain & 0.80 & -1.01 \\
\hline Asthma & 0.63 & -0.18 \\
\hline Thyroid problems & 0.28 & -0.26 \\
\hline Eye disease & -0.05 & 0.71 \\
\hline Narrowing of bloodvessels & -0.26 & -1.18 \\
\hline Coronary heart disease & -0.35 & -1.43 \\
\hline
\end{tabular}

The contribution of chronic conditions to the attributable absenteeism cost of excess weight was computed with the same methodology applied for the direct health care costs. 13 chronic diseases had a positive contribution to the incremental cost of absenteeism (Appendix Table 10). Arthritis, including rheumatoid arthritis and osteoarthritis, was the most important driver of the incremental cost of absenteeism in overweight and obese individuals, followed by hypertension and low back pain.

\section{Discussion}

This study aimed to evaluate the societal impact of excess weight by estimating the direct and indirect costs associated with overweight and obesity among adults in Belgium. Our findings are based on a linkage of national health survey and health reimbursed cost data in Belgium for the years 2013 to 2017.

Average yearly health care costs attributed to overweight and obese individuals were significantly higher (i.e. $43 \%$ and $77 \%$ higher) than average costs among normal weight individuals. When adjusting for age, gender, household educational level and the lack of physical activity, the cost gap was reduced to $24 \%$ and $36 \%$ for respectively the population with overweight and obesity. Regarding the costs of absenteeism, individuals with obesity had a significantly higher cost compared to people with a normal weight ( $87 \%$ higher). Our results showed that in Belgium approximately €3.3 billion is spent yearly on average for direct healthcare costs due to excess body weight. It represents approximately $13.5 \%$ of the total yearly healthcare costs in Belgium and $10 \%$ of the yearly budget reserved to healthcare [19]. Yearly productivity loss due to work absenteeism poses an average cost of $€ 1.2$ billion that could be attributed to overweight and obesity in the Belgian working population.

In line with our estimates, OECD showed that the average healthcare expenditure for an obese person is $25 \%$ higher than for someone of normal weight [20]. Moreover, it is estimated that $€ 70$ billion are spent annually in Europe for healthcare and productivity loss due to obesity [21]. Other countries performed analysis similar to ours. Veiga (2008) compared two waves of the Portuguese National Health Survey (1996 vs 1999). Between the two waves, the total health care expenditures almost tripled for overweight people (€133 vs €366 million) and more than doubled for people with obesity (€124 vs €261 million) [22]. Emery et al. (2007) estimated direct healthcare costs of obesity in France to be between $€ 2.1$ and $€ 6.2$ billion based on the Survey on Health and Social Protection of 2002 [23]. 
Considering that high $\mathrm{BMI}$ is associated with increased comorbidity, contributing to an increase in costs, we also investigated the relative contribution of different chronic diseases to the cost attributable to excessive body weight. In our study, hypertension constitutes by far the major contributor to incremental costs due to excess weight, followed by high cholesterol and serious gloom or depression. Different type of arthritis formed the main comorbidity driving the costs related to absenteeism, followed by hypertension and low back pain.

In a study conducted in the US looking at electronic medical records and claims, hypertensive diseases, dyslipidaemia, and osteoarthritis were the three most expensive obesity-related comorbidities at the population level; each responsible for \$18 million annually. Moreover, it was found that hypertension and osteoarthritis were much more costly among individuals with obesity than those without obesity [24]. In Padula et al (2014), total net expenditures of obesity and its comorbidities were calculated based on US claims in 2012. The combination of obesity and hypertension was the most common condition (inpatient and outpatient claims) accounting for a mean total cost of around $\$ 4,000$, followed by obesity and diabetes and obesity and depression [25].

Our study provides valuable information on the extent of the societal impact that excessive weight status has in Belgium. The approach of recycled predictions has allowed us to compare direct and indirect healthcare costs among different BMI categories while adjusting for confounding by including important sociodemographic and health status covariates in the models. Our findings are also important from a health policy perspective, in the planning of strategies for health care cost containment. From a public health perspective, a sustainable approach towards effective prevention of the most impactful diseases is a more affordable strategy [26]. Public health programs to promote weight reduction and weight management among obese and overweight people play an important role in curbing the economic burden of different diseases. According to the state of health report of the EU countries, there are many modifiable behavioural risk factors related to overweight and obesity that could be improved. In Belgium, about $25 \%$ of people do not eat any vegetables and $45 \%$ any fruit daily. Moreover, Belgian adults are less physically active than those in many EU countries [27] and on average one third of their consumption is from ultra-processed food products [28].

We acknowledge some limitations within our study. First, there are some limitations that are intrinsic of the nature of our data sources. Self-reported data, deriving from national surveys, is subject to recalling and social desirability biases. This might have influenced primarily the reporting of height and weight, known to be a source of underestimation within the BHIS [29], as well as the amount of non-responses for heavy daily smoking and lack of physical activity that led to a considerable reduction of the sample size. In addition, participants with a low socio-economic status are more likely to leave questions without answering [30] and to be subject to excess weight status [31]. This might have led to underestimation of the prevalence of overweight and obesity. Nevertheless, surveys represent an essential source of information for lifestyle characteristics, like smoking, eating habits, and chronic diseases that remain frequently un-diagnosed so they are difficult to grasp with other types of data sources (e.g. low back pain). With regard to cost data, national claims data collected at population-level do not include services that are not covered by the insurance (e.g. ambulant psychotherapy, limited reimbursements for physiotherapy). Even so, administrative data are an essential source for investigating the financial burden of healthcare. A further limitation is the possibility of residual confounding bias. We tried to overcome this by increasing the chance of detecting measured confounders via the double-selection process, but it may well be that certain important confounders were lacking from the database. The analysis of the relative contribution of diseases is especially vulnerable to this, as it additionally needs adjustment for common causes of disease and health care costs, and ignores that the considered diseases may mutually influence each other. In addition, some variables suffered from a high rate of non-responses, decreasing the sample size and possibly introducing bias. Nevertheless, comparing the socio-demographic characteristics of the initial sample and those of the reduced one showed no particular difference (Appendix Table 6). In future analyses, multiple imputation could be used for addressing potential selection bias and for lessening the information loss that results from the reduced sample size. Authors are also aware that in the observed 5-years after filling in the survey might have lost weight and change BMI status. Nevertheless, we were interested in looking in the long term chronic effects of excess weight, that is why we were interested in having a follow-up as long as possible. This limitation highlights the need and importance of cohort studies that allow to follow-up participants through time. 
Table 6

Socio-demographic characteristics by body mass index category, Belgian population $\geq 18$ years, health interview survey 2013 for population included in the multivariate regression (with no missing values in physical activity and educational

\begin{tabular}{|c|c|c|c|c|c|c|c|c|c|c|}
\hline & \multicolumn{2}{|l|}{ Total } & \multicolumn{2}{|c|}{ Underweight } & \multicolumn{2}{|c|}{ Normal weight } & \multicolumn{2}{|c|}{ Overweight } & \multicolumn{2}{|c|}{ Obese } \\
\hline & $\mathrm{N}^{(1)}$ & $\%^{(2)}$ & $\mathrm{N}$ & $\%$ & $\mathrm{~N}$ & $\%$ & $\mathrm{~N}$ & $\%$ & $\mathrm{~N}$ & $\%$ \\
\hline Total & 4,624 & 100 & 120 & 2.5 & 2,262 & 49.1 & 1,600 & 35.5 & 642 & 12.9 \\
\hline \multicolumn{11}{|l|}{ Gender } \\
\hline Men & 2,178 & 48.1 & 21 & 17.9 & 923 & 42.4 & 942 & 58.7 & 292 & 44.8 \\
\hline Women & 2,446 & 51.9 & 99 & 82.1 & 1,339 & 57.7 & 658 & 44.8 & 350 & 55.2 \\
\hline \multicolumn{11}{|l|}{ Age } \\
\hline $18-34$ years & 1,135 & 25.3 & 61 & 62.7 & 715 & 30.5 & 272 & 18.4 & 87 & 14.4 \\
\hline $35-65$ years & 2,458 & 54.4 & 41 & 26.3 & 1,140 & 53.0 & 892 & 55.9 & 385 & 61.1 \\
\hline$\geq 66$ years & 1,031 & 20.3 & 18 & 11.0 & 407 & 16.5 & 436 & 25.8 & 170 & 24.5 \\
\hline \multicolumn{11}{|l|}{ Household education } \\
\hline No diploma/primary & 342 & 7.1 & 6 & 1.9 & 100 & 3.8 & 151 & 9.4 & 85 & 15.4 \\
\hline Lower secondary & 596 & 12.3 & 15 & 15.1 & 242 & 9.8 & 221 & 13.2 & 118 & 17.8 \\
\hline Higher secondary & 1,454 & 33.1 & 36 & 37.7 & 683 & 33.0 & 508 & 32.6 & 227 & 34.4 \\
\hline Higher education & 2,232 & 47.5 & 63 & 45.3 & 1,237 & 53.4 & 720 & 44.9 & 212 & 32.5 \\
\hline \multicolumn{11}{|l|}{ Household income } \\
\hline Quintile 1 & 607 & 12.3 & 23 & & 258 & & 204 & & 122 & \\
\hline Quintile 2 & 630 & 14.3 & 17 & & 277 & & 233 & & 103 & \\
\hline Quintile 3 & 875 & 20.9 & 21 & & 419 & & 302 & & 133 & \\
\hline Quintile 4 & 979 & 24.7 & 28 & & 508 & & 331 & & 112 & \\
\hline Quintile 5 & 1,127 & 27.8 & 7 & & 199 & & 140 & & 60 & \\
\hline \multicolumn{11}{|c|}{ Number of chronic conditions } \\
\hline None & 2,887 & 63.9 & 88 & 76.4 & 1,634 & & 899 & 57.4 & 266 & 41.6 \\
\hline 1 & 1,121 & 23.5 & 17 & 16.1 & 453 & & 457 & 26.9 & 194 & 31.2 \\
\hline 2 & 455 & 9.4 & 12 & 6.7 & 137 & & 184 & 12.1 & 122 & 19.4 \\
\hline 3 or more & 160 & 3.1 & 3 & 0.7 & 37 & & 60 & 3.5 & 60 & 7.8 \\
\hline
\end{tabular}

Considering that there is currently no national nutrition and physical activity health plan in Belgium [32], our estimates can inform policy makers and ease evidence-based interventions. In 2019, the WaIST project was initiated in Belgium aiming to provide proactive policy support for the prevention of excessive weight gain [33]. As part of this project health impact assessment will be used to model different internationally recommended health policies tackling overweight and obesity. Acting on the risk factors will help to reduce a cumbersome burden carried by our society largely affected by non-communicable diseases.

\section{Conclusions}

Based on national health and financial estimates, we found that high BMI has a substantial societal economic burden in Belgium. We estimated that every year at least $€ 4.5$ billion are spent to cover the direct and indirect costs related to overweight and obesity. Policies and interventions are urgently needed to reduce the prevalence of overweight and obesity thereby decreasing these substantial costs.

\section{Abbreviations}

BHES: Belgian health examination survey

BHIS: Belgian health interview survey

BMI: body mass index

Cl: confidence interval 
DALY: Disability-adjusted life years

IMA: Intermutualistic agency

RR: Risk ratio

\section{Declarations}

Ethics approval and consent to participate

Not applicable

Consent for publication

Not applicable

Availability of data and materials

All data generated during this study is included in the appendix of this article.

Competing interests

The authors declare that they have no competing interests.

Funding

VG received funds in the context of this work by Sciensano, through the WaIST project. The organization of the Belgian National Health Interview Survey was funded by the federal, regional and community health authorities in Belgium. The linkage of the survey data with the health insurance data was funded by the National Institute for Health and Disability Insurance Belgium.

Authors' contributions

$\mathrm{BD}$ and IM designed the model and the computational framework. IM and VG carried out the implementation and performed the calculations, under the supervision of SV and BD. IM and VG wrote the manuscript with input from all authors. All the authors approved the manuscript.

Acknowledgements

The authors thank Stephan Devriese from the Belgian healthcare knowledge center (KCE) for his help in the interpretation and adaptation of cost data.

\section{References}

1. L. M. Jaacks et al., "The obesity transition: stages of the global epidemic," Lancet Diabetes Endocrinol., vol. 7, no. 3, pp. 231-240, Mar. 2019, doi: 10.1016/S2213-8587(19)30026-9.

2. T. Vos et al., "Global burden of 369 diseases and injuries in 204 countries and territories, 1990-2019: a systematic analysis for the Global Burden of Disease Study 2019," The Lancet, vol. 396, no. 10258, pp. 1204-1222, Oct. 2020, doi: 10.1016/S0140-6736(20)30925-9.

3. R. Kelishadi, "Health impacts of Obesity," Pak. J. Med. Sci., vol. 31, no. 1, Nov. 2014, doi: 10.12669/pjms.311.7033.

4. S. Kent, F. Fusco, A. Gray, S. A. Jebb, B. J. Cairns, and B. Mihaylova, "Body mass index and healthcare costs: a systematic literature review of individual participant data studies: BMI and healthcare costs," Obes. Rev., vol. 18, no. 8, pp. 869-879, Aug. 2017, doi: 10.1111/obr.12560.

5. A. Goettler, A. Grosse, and D. Sonntag, "Productivity loss due to overweight and obesity: a systematic review of indirect costs," BMJ Open, vol. 7, no. 10, p. e014632, Oct. 2017, doi: 10.1136/bmjopen-2016-014632.

6. OECD, The Heavy Burden of Obesity: The Economics of Prevention. OECD, 2019. doi: 10.1787/67450d67-en.

7. A. M. Bender, J. Sørensen, F. Diderichsen, and H. Brønnum-Hansen, "A health inequality impact assessment from reduction in overweight and obesity," BMC Public Health, vol. 20, no. 1, p. 1823, Dec. 2020, doi: 10.1186/s12889-020-09831-x.

8. "Health Interview Survey," Sciensano, Bruxelles, Belgium, D/2019/14.440/62. [Online]. Available: https://his.wivisp.be/fr/Documents\%20partages/NS_FR_2018.pdf

9. L. Annemans, “De prijs van uw gezondheid. Is onze gezondheid in gevaar?," Tielt, 2014.

10. N. Verhaeghe, O. De Greve, and L. Annemans, "The potential health and economic effect of a Body Mass Index decrease in the overweight and obese population in Belgium," Public Health, vol. 134, pp. 26-33, May 2016, doi: 10.1016/j.puhe.2016.01.015.

11. S. Demarest, J. Van der Heyden, R. Charafeddine, S. Drieskens, L. Gisle, and J. Tafforeau, "Methodological basics and evolution of the Belgian health interview survey 1997-2008," Arch. Public Health, vol. 71, no. 1, p. 24, Dec. 2013, doi: 10.1186/0778-7367-71-24.

12. WHO, "Body mass index - BMI." https://www.euro.who.int/en/health-topics/disease-prevention/nutrition/a-healthy-lifestyle/body-mass-index-bmi (accessed Jun. 14, 2021).

13. R Core Team, R: A language and environment for statistical computing. Vienna, Austria: R Foundation for Statistical Computing, 2021. [Online]. Available: https://www.R-project.org/

Page $17 / 19$ 
14. O. Urminsky, C. Hansen, and V. Chernozhukov, “The Double-Lasso Method for Principled Variable Selection.,” PsyArXiv, preprint, Sep. 2019. doi: 10.31234/osf.io/2pema.

15. N. Keiding and D. Clayton, "Standardization and Control for Confounding in Observational Studies: A Historical Perspective," Stat. Sci., vol. 29, no. 4, Nov. 2014, doi: 10.1214/13-STS453.

16. J. M. Snowden, S. Rose, and K. M. Mortimer, "Implementation of G-computation on a simulated data set: demonstration of a causal inference technique," Am. J. Epidemiol., vol. 173, no. 7, pp. 731-738, Apr. 2011, doi: 10.1093/aje/kwq472.

17. STATBEL, "Population by place of residence, nationality (Belgian/non-Belgian), marital status, age and gender," Jun. 16, 2021. https://bestat.statbel.fgov.be/bestat/crosstable.xhtml?datasource=65ee413b-3859-4c6f-a847-09b631766fa7 (accessed Jun. 24, 2021).

18. Cleemput Irina, Neyt Mattias, Van De Sande Stefaan, and Thiry Nancy, "Belgian guidelines for economic evaluations and budget impact analyses: second edition," Belgian Health Care Knowledge Centre (KCE), Brussels, Health Technology Assessment (HTA) 183C, 2012.

19. Verbond van Belgische Ondernemingern, "BEGROTING GEZONDHEIDSZORG VOOR 2021 GOEDGEKEURD IN RIZIV."

20. OECD, Obesity and the Economics of Prevention: Fit not Fat. OECD, 2010. doi: 10.1787/9789264084865-en.

21. F. Erixon, “Europe's Obesity Challenge," European Centre for International Political Economy, 2016. Accessed: Jun. 14, 2021. [Online]. Available: http://www.jstor.org/stable/resrep23968

22. P. Veiga, "Out-of-pocket health care expenditures due to excess of body weight in Portugal," Econ. Hum. Biol., vol. 6, no. 1, pp. 127-142, Mar. 2008, doi: 10.1016/j.ehb.2007.08.001.

23. C. Emery, J. Dinet, A. Lafuma, C. Sermet, B. Khoshnood, and F. Fagnani, “[Cost of obesity in France]," Presse Medicale Paris Fr. 1983, vol. 36 , no. 6 Pt 1, pp. 832-840, Jun. 2007, doi: 10.1016/j.lpm.2006.12.031.

24. Q. Li, S. W. Blume, J. C. Huang, M. Hammer, and M. L. Ganz, "Prevalence and healthcare costs of obesity-related comorbidities: evidence from an electronic medical records system in the United States," J. Med. Econ., vol. 18, no. 12, pp. 1020-1028, Dec. 2015, doi: 10.3111/13696998.2015.1067623.

25. W. V. Padula, R. R. Allen, and K. V. Nair, "Determining the cost of obesity and its common comorbidities from a commercial claims database: Cost of obesity and comorbidities," Clin. Obes., vol. 4, no. 1, pp. 53-58, Feb. 2014, doi: 10.1111/cob.12041.

26. J. C. Seidell and J. Halberstadt, "The global burden of obesity and the challenges of prevention," Ann. Nutr. Metab., vol. 66 Suppl 2, pp. 7-12, 2015, doi: $10.1159 / 000375143$.

27. Organisation de coopération et de développement économiques and European Observatory on Health Systems and Policies, Belgium: Country Health Profile 2019. 2019. Accessed: Apr. 27, 2021. [Online]. Available: https://doi.org/10.1787/3bcb6b04-en

28. S. Vandevijvere, K. De Ridder, T. Fiolet, S. Bel, and J. Tafforeau, "Consumption of ultra-processed food products and diet quality among children, adolescents and adults in Belgium," Eur. J. Nutr., vol. 58, no. 8, pp. 3267-3278, Dec. 2019, doi: 10.1007/s00394-018-1870-3.

29. S. Drieskens, S. Demarest, S. Bel, K. De Ridder, and J. Tafforeau, "Correction of self-reported BMI based on objective measurements: a Belgian experience," Arch. Public Health, vol. 76, no. 1, p. 10, Dec. 2018, doi: 10.1186/s13690-018-0255-7.

30. T. Tsiampalis and D. B. Panagiotakos, "Missing-data analysis: socio- demographic, clinical and lifestyle determinants of low response rate on selfreported psychological and nutrition related multi- item instruments in the context of the ATTICA epidemiological study," BMC Med. Res. Methodol., vol. 20, no. 1, p. 148, Dec. 2020, doi: 10.1186/s12874-020-01038-3.

31. B. Loring, A. Robertson, Organisation mondiale de la santé, and Bureau régional de l'Europe, Obesity and inequities: guidance for addressing inequities in overweight and obesity. Copenhagen, Denmark: World Health Organization, Regional Office for Europe, 2014.

32. Sciensano, "WaIST - Contribution of excessive weight status to the social impact of non-communicable diseases, multimorbidity and disability in Belgium: past, present, and future." https://www.sciensano.be/en/projects/contribution-excessive-weight-status-social-impact-non-communicablediseases-multimorbidity-and (accessed Jun. 18, 2021).

33. V. Gorasso et al., "Impact of overweight on the burden of non-communicable diseases in Belgium: the WalST project," Eur. J. Public Health, vol. 30 , no. Supplement_5, p. ckaa166.412, Sep. 2020, doi: 10.1093/eurpub/ckaa166.412.

\section{Figures}



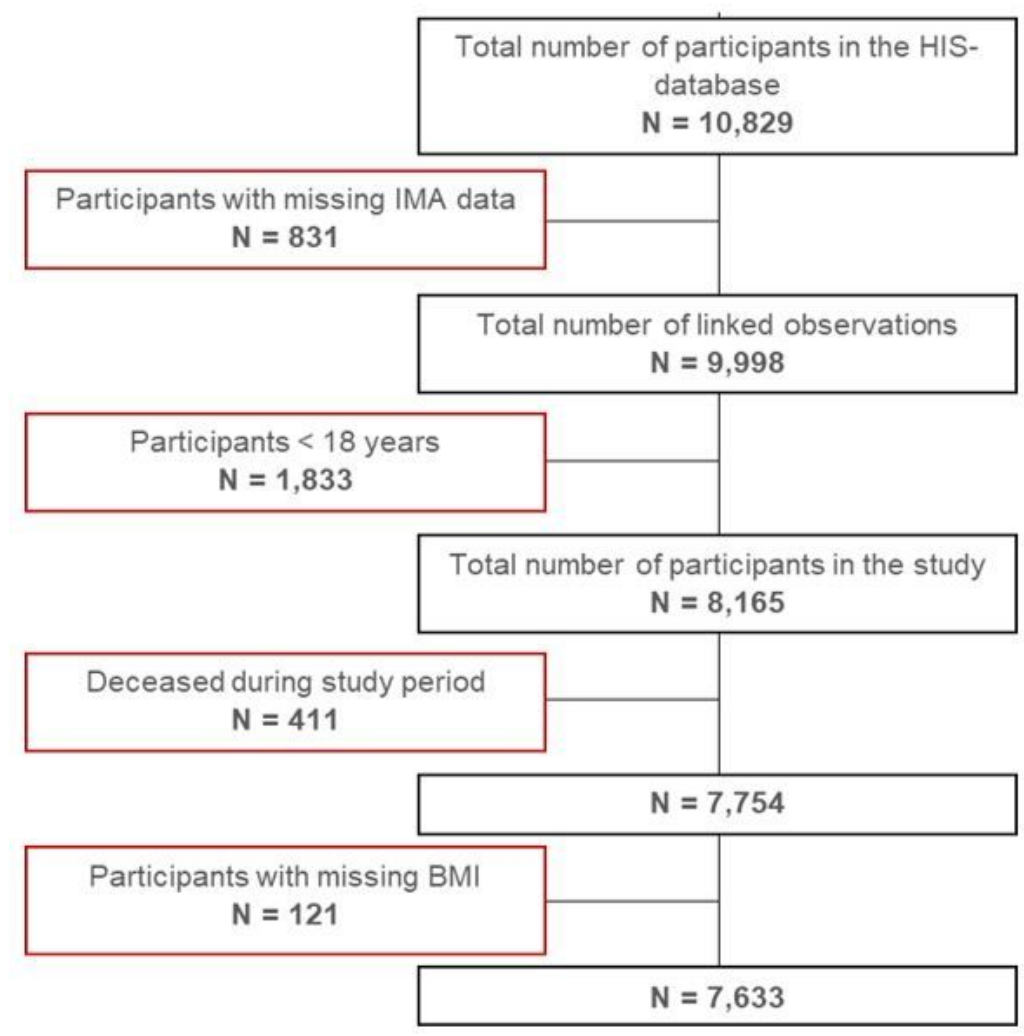

Figure 1

Flow diagram of number of respondents included in the study

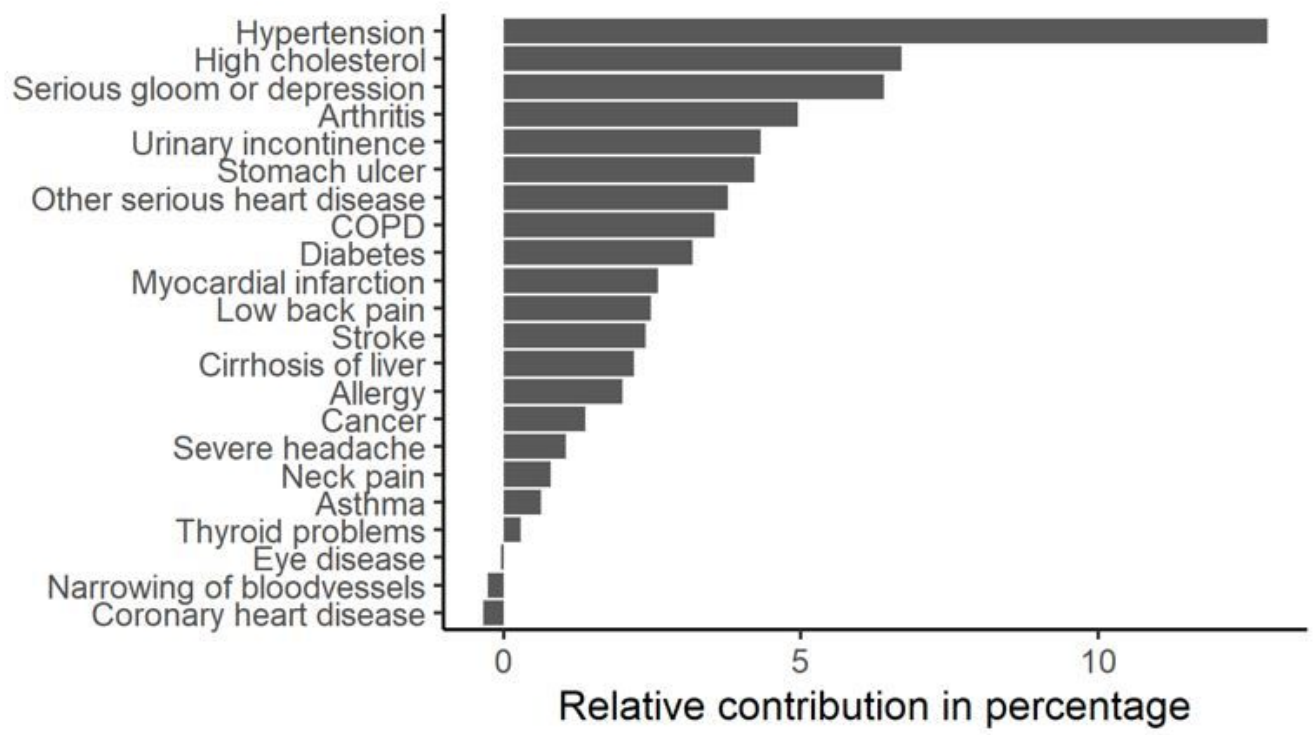

Figure 2

Relative contribution of chronic conditions to incremental costs of overweight and obesity, Belgian population $\geq 18$ years , BHIS 2013-IMA2013-2017 